\title{
Die beiden Keimblätter und der Mittelkeim.
}

Von

\author{
Dr. W. Wolff in Berlin.
}

Hierzu Tafel XXVII.

Der erste Forscher, der die Zusammensetzung des Keimes aus blattartigen Schichten nachwies, war Pander und zwar unterscheidet derselbe an dem Keime des Huhnes erst nur eine Schicht zusammenhängender Körner, das Schleimblatt, an dessen Aussenseite alsbald eine dünnere durchsichtige Lage, das seröse Blatt entsteht, zwischen diesem bildet sich seine dritte Lage, die Gefässschicht. Van Baer ging einen Schritt weiter, indem er fand, dass sich die mittlere hlattförmige Anlage wiederum in zwei Blätter theilte, von denen er das äussere Fleischschicht nannte und mit dem oberen Blatte, dem Hautblatte, zusammen als animale Anlage bezeichnete; das innere nannte er Gefässschicht und bezeichnete es mit dem unteren Blatte, dem Schleimblatte zusammen als vegetative Anlage. Von Remak an kann man eine neue Epoche in der Deutung der blattförmigen Embryonalanlagen rechnen, die für die Auffassung der Entwicklung der gesammten Metazoen von Bedeutung war. Remak beschreibt die Entstehung der blattförmigen Embryonalanlagen beim Huhne rom Zeitpunkte des gelegten Eies an und zwar besteht nach ihm der Keim des frisch gelegten Eies aus einem fester gefügten äusseren Blatte, dem äusseren Keimblatte, und einer loser gefügten inneren Schicht, die er inneres Keimblatt nannte. In Folge der Bebrutung scheidet sich vom inneren Keimblatte eine dritte blattartige Anlage, das Darmdrüisenblatt ab, den Rest des inneren Keimblattes bezeichnet Remak als mittleres Keimblatt; so ist das innere Keimblatt in das mittlere und in das Darmdruisenblatt aufgegangen. Ferner beschreibt er, wie das mittlere Keimblatt nun in strichförmiger Ausdehnung im Primitivstreifen mit dem äusseren Keimblatte ver- 
schmilzt und schliesst aus guten Grïnden, dass in dieser Vereinigung der beiden Keimblätter böchst wahrscheinlich ein Austausch der Elemente stattinde; den bestimmten Nachweis konnte er nicht führen, da die mikroskopische Technik noch nicht soweit vorgeschritten war, dass man den Embryo in zusammenhängenden Schnittreihen untersuchte.

Die thatsächlichen Befunde Remak's sind vollstïndig richtig, dagegen ist seine Auffassung derselben und die damit in Zusammenhang stehende Bezeichnung eine leider irrthiimliche gewesen und ist vielleicht mit ein Grund, dass die Verwirrung in der Auffassung und Dentung der Keimblätter jetzt eine so grosse geworden ist, dass es z. B. bei den Wirbelthieren keine Combination fiir die Entstehung des mittleren Keimblattes giebt, die nicht ihre Vertreter hätte. So hat die Ansicht, dass das mittlere Keimblatt aus dem äusseren, wie aus dem inneren, wie aus beiden, wie endlich aus keinem von beiden Keimblättern entstanden sein soll, ihre Vertreter unter den Forschern.

Ein Fortschritt war es, dass Re mak den Namen „Keimblatt“ für die primitiven embryonalen blattförmigen Anlagen und deren verschiedene Bezeichnungen einführte; durch diesen Namen war die Verwandtschaft der Blätter unter sich angedeutet, und es war eine berechtigte Forderung, dass man unter diesem Namen gleichwerthige Grössen zusammenfasste, die auch womöglich auf eine homologe Weise sich gebildet hätten, und zwar, wie der Name Keimblatt andeutete, als blattartige Anlagen aus dem Keime. Der Fehler der Remak'schen Auffassung und Bezeichnung liegt darin, dass er den Theil des Keimes, der nach Abzug des äusseren Keimblattes übrig bleibt, inneres Keimblatt nannte, obgleich dieser Theil des Keimes überhaupt noch gar keine blattförmige Anordnung zeigt, was auch $\mathrm{Remak}$ wohl bekannt war und aus seiner Beschreibung dieses Theiles des Keines klar hervorgeht. Die Folge nun, die aus dieser Benennung resultirt, ist die, dass alle weiteren Differenzirungen dieses Theiles des Keimes als aus dem inneren Keimblatte entstanden aufgefasst werden müssen, und doch bildet sich das wirkliche innere Keimblatt erst aus diesem Theile des Keimes. Ein anderer Febler dieser Benennung liegt darin, dass, nachdem das innere Keimblatt Remak's in das mittlere und das Darmdritsenblatt aufgegangen ist, jetzt der Keim aus einem äusseren und einem mittleren Keimblatte bestehen soll, ohne ein inneres zu 
besitzen. Letztere Inconsequenz machte sich allerdings bald fühlbar, obgleich dies nirgends klal ausgesprochen ist, und wurde von den Forschern alsbald in soweit ausgeglichen, als dieselben von dem inneren Keimblatte $R$ emak's, das sie primäres inneres Keimblatt oder inneres Keimblatt im weiteren Sinne nannten, sich das mittlere als einen Theil desselben abspalten liessen, und nun den Namen secundäres inneres Keimblatt oder inneres Keimblatt im engeren Sinne anf den übrig bleibenden Rest übertrugen, den Remak als Darmdrïsenblatt hezeichnet hatte. Dieser Auffassung huldigen die meisten Forscher ${ }^{1}$ ) noch heute und doch liegt der Fehler derselben auf der Hand: man stellt nämlich zwei gleichzeitige und gleichwerthige Bildungen so dar, als wäre die eine aus der anderen entstanden.

Für Remak hatte die Benennung der blattartigen embryonalen Zellschichten noch nicht die Bedeutung, die wir derselben heute beilegen müssen, da sich inzwischen herausgestellt hat, dass die Keimblätter Bildungen sind, die wir bei den gesammten Metazoen finden; wir können dieselben jedoch nur dann mit einander vergleichen, wenn wir homolog entstandene Bildungen mit diesem Namen bezeichnen und nicht jede beliebige blattförmige Anlage im Embryo Keimblatt nennen. Dazu kommt noch der Umstand, dass bei den niedrigen Classen der Metazoen überhaupt nur zwei Keimblätter vorhanden sind, wir also genau prïfen müssen, ob einer bei den höheren Classen der Metazoen neu auftretenden dritten Schicht auch der Werth und die Bedeutung eines Keimblattes zukommt. Wir mïssen uns also vor allem genau darüber verständigen, was wir als Keimblatt bezeichnen wollen und dürfen. Aus diesem Grunde werde ich erst kurz die allgemeine Bildung der Keimblätter der Metazoen recapituliren. Hierans werden sich dann die den Keimblättern besonders eigenthümlichen Momente ergeben. Da aber die Keimblattbildung ihren Ursprung der Gastrula-

1) So lässt Balfour in seinem jüngst erschienenen Werke der vergleichenden Embyologie durchgängig den Vesoblast aus dem primitiven Hypoblast entstehen. Auch die Stricker'sche Schule, Peremuschko etc. machen hiervon keine Ausnahme, denn wenn dieselben vom Boden der Keimhöhle Zellen $\mathbf{z w i s c h e n}$ die beiden primitiven Keimblätter wandern lassen, und aus diesen Zellen das mittlere Keimblatt entstehen soll, so geht es ebenfalls aus dem primitiven Hypoblast hervor. 
bildung verdankt, die Form der Gastrula wiederum zum Theil abhängig von der Beschaffenheit des Eies ist, so lasse ich hier die Entstehung der verschiedenen Eiformen und die Wechselbeziehungen zwischen Ei und Gastrula, wie sie auf Taf. XXVII, Fig. 1 bis 7 graphisch dargestellt sind, folgen. Voran stelle ich einige Voraussetzungen, auf welche sich die weiter unten folgenden Schlüsse aufbauen und die wohl kaum einen Widerspruch finden werden:

Die ursprüngliche Form des primitiven Eies im Ruhezustand ist die Kugelform, die das Ei auch trotz verschiedener Veränderungen in Inneren stets wieder anzustreben sucht; es sind hier Veränderungen gemeint, die durch die grösseren Anforderungen bei höherer Entwicklung des zukünftigen Organismus schon an das Ei gestellt werden.

Der Kern der Zelle, in unserem Falle das Keimbläschen, ist durch bestimmte Kräfte nach bestehenden Gesetzen an seiner Stelle fixirt. Aus der Betrachtung der Eier der niedersten Organismen und der jungen Eier zu schliessen, scheint die Lage des Keimbläschens in der Mitte des Bildungsdotters, also ursprünglich im Centrum des Eies zu sein.

Ferner können wir aus der Kenntniss der Entwicklung verschiedener Eier, so namentlich der Amphibieneier, als Gesetz hinstellen, dass im befruchteten $\mathrm{Ei}$ das Protoplasma sich möglichst so lagert, dass die Zelle, die aus einer bestimmten Stelle dieses Protoplasmas durch die Furchung hervorgeht, auch sofort an dem ihr wenigstens vorläufig zukommenden Platz oder ihrem Bestimmungsort thunlichst nahe sich befindet.

Fig. 1 stellt ein primitives Ei vor, die untere Horizontale theilt den Bildungsdotter sämmtlicher Eier ohne Rïcksicht auf die oben angedeuteten späteren Veränderungen derselben in zwei Hälften und stellt zugleich die erste horizontale Furchungsebene vor. Die Vertikale theilt ebenfalls den Bildungsdotter in zwei Hälften ohne Rücksicht auf spätere Veränderungen und stellt die erste vertikale Furchungsebene dar. Beide Ebenen gehen nach der obigen Voraussetzung selbstverständlich durch den Kern. Da bei dieser Theilung ein oberer und unterer Eipol vorausgesetzt ist, so mag hier erwähnt werden, dass der obere Pol bei unbehinderter Beweglichkeit des Eies der dem Lichte zugekehrte ist.

Durch die Furchung der Fig. 1 a entsteht die Blastula Fig. $1 \mathrm{~b}$ 
und aus ihr durch Einstïlpung der unteren Hälfte gegen die obere, durch embolische Invagination, die Gastrula.

An der Gastruba unterscheiden wir bekanntlich eine äussere Zellschicht, das äussere Keimblatt, eine innere Zellschicht, das innere Keimblatt, die Höhle, das Archenteron und die Verbindung: derselben mit der Aussenwelt, den Blastogorus oder das Prostoma.

Wenn nun das Ei durch den länger dauernden Entwicklungsprocess des Embryo gezwungen wird, Nahrung im Vorrath mitzunehmen, so ist es natürlich, dass dieselbe in dem Theile des Eies deponirt wird, der im Blastulastadium dem späteren inneren Blatte der Gastrula entspricht resp. ihm am nächsten gelegen ist, weil dieser Theil ansschliesslich die Aufgabe der Ernälurung des Organismus ubernommen hat. Auf diese Weise entsteht ein Ei, wie es schematisch in Fig. 2 wiedergegeben ist und in Fig. 2 a gezeichnet ist, nachdem es wieder die Kugelform angenommen hat, An solchen Eiern kann man den Bildungspol, der mit dem oberen Pol libereinstimmt, von dem Dotterpol (unteren Pol) unterscheiden und zwar nimmt der Bildungsdotter mit der Entfernung von seinem Pole in dem Verhältnisse ab, wie der Nabrungsdotter zunimmt. Wahrscheinlich steht die Linsenform des Keimbläschens auch mit der Dotteraufnahme des Eies im Zusammenhange, insofern nämlich durch dieselben das ursprüngliche kugelförmige Keimbläschen zusammengedrückt wird. Ferner muss das Keimbläschen, wenn es seinen Standort in der Mitte des Bildungsdotters beibehalten soll, dem oberen Pole näher rücken. Durch diese Ortsveränderung, die das Keimbläschen dem ganzen Ei gegenüber annimmt, wird die inäquale Furchung bedingt, insofern die erste horizontale Furchungsebene, die auch die vorhandenen (gewöhnlich vier Kerne) trifft, wohl den Bildungsdotter halbirt, das Ei jedoch in zwei ungleiche Stücke theilt. Ist die Nahrungsdotteraufnahme des Eies noch keine allzu grosse, so uibt sie keinen Einfluss auf die Art der Gastrulabildung aus, es wird nur, wie Fig. 2 c zeigt, die archentorische Höhle kleiner, und überwiegen, wie schon im Blastulastadium zu sehen ist, die unteren Zellen an Masse bei weitem die oberen. Fig. 2 b.

Die ersten Differenzirungen der Gastrula bestehen bekanntlich in der Abflachung der einen Seite und in der Bildung des Kopfes (Mundes) gegenuber dem Blastoporus. Diese beiden Momente haben jedoch keine Rückwirkung auf die Eiform, dagegen eine 
weitere Veränderung, die bei den Wirbelthieren auftritt, nämlich die ausserordentliche Ausbildung des unteren Rückenendes, die bei den niederen Wirbelthieren in der Form des Schwanzes, bei den höheren unter Rückbildung des Schwanzes in der Form der hinteren Extremitäten sich darbietet. Die Folge biervon ist eine gesteigerte Anforderung von Bildungsmaterial an das untere Riickenende der Gastrula. Die Gastrula stellt diese Forderung wiederum an den dieser Stelle entsprechenden Theil der Blastula und diese an das Ei. Es entsteht so durch besonderes Wachsthumsbedürfuiss einer bestimmten Stelle des Eies die asymmetrische Anhäufung des Bildungsdotters um das Keimbläschen (das, wie vorausgesandt, an einer bestimmten Stelle des primitiven Eies fixirt gedacht werden muss), wie sie schematisch in Fig. 3 gezeichnet ist und in. Fig. 3 a, nachdem das $\mathrm{Ei}$ wiederum die Kugelform erreicht bat. Diese asymmetrische Anbäufung des Bildungsdotters um das Keimbläschen findet ihren Ausdruck in der asymmetrischen Furchung insofern die Furchungsebene durch den Kern gehen muss. $\mathrm{Ob}$ und in wieweit die asymmetrische Anhäufung des Bildungsdotters um den Kern speciell auch auf Rechnung des Mittelkeims, von dem weiter unten die Rede sein wird, gesetzt werden darf, wird von dem ev. sicheren Nachweis einer asymmetrischen Furchung bei Eiern wirbelloser Thiere abhängen. Die asymmetrische Furchung ist wohl von der inäqualen Furchung zu unterscheiden, denn letztere beruht, wie oben auseinandergesetzt, auf der Aufnahme von Nahrungsmaterial in das Protoplasma der Zelle, findet gegenüber der asymmetrischen Furchung ihren Ausdruck in der Grösse ihrer Theilungsendproducte und bezieht sich auf die beiden Theile des Eies, die durch eine auf der Verbindungsebene des Bildungspol mit dem Dotterpol senkrecht stehenden Ebene gebildet werden. Die asymmetrische Furchung, auf die zuerst $\mathrm{K} \ddot{b l l i k e r}$ beim Hühnchen aufmerksam gemacht hat, findet sich selbstverständlich auch bei den Reptilien und Fisch-Eiern. Bei den -Amphibieneiern, bei denen meines Wissens nach diese Asymmetrie bisher nicht beachtet wurde, kann man dieselbe ebenfalls sebr deutlich sehen. Es geht nämlich die erste Furchungsebene nicht durch die Mitte des Eies, sondern theilt dasselbe in zwei ungleiche Hälften, die dritte Furchungsebene (erste horizontale Ebene) steht ausserdem nicht rechtwinklig zu den beiden vertikalen, sondern macht einen stumpfen Winkel mit der ersten vertikalen an der Seite der 
grösseren Hälfte. In wieweit die als nicht ganz regulär beschriebene Furchung der Säugethiereier auf eine asymmetrische Furchung, anstatt, wie bisher, auf eine inäquale zu beziehen ist, mïssen spätere auf diesen Punkt gerichtete Untersuchungen lehren.

Die Asymmetrie der Furchung in Verbindung mit der steigenden Zunahme an Nabrungsdotter des Eies geben der Blastula schon eine ganz veränderte Form. Fig. $3 \mathrm{~b}$ zeigt das Schema einer solchen Blastula. Die Blastulahöhle ist durch die ausserordentlicbe Aufnahme von Nahrungsdotter der unteren Zellen sehr verkleinert worden. Das Auffallendste jedoch an einer solchen Bastula ist die Asymmetrie der beiden oberen Hälften, die Anhäufung von Bildungsmaterial an einem bestimmten Theile der Blastula. Stülpt sich nun der durch die Blastulahöhle (Keimhöhle) getrennte untere Theil gegen den oberen ein, so liegt es auf der Hand, dass die Seite mit dem überwiegenden Bildungsmaterial auch einen grösseren Theil der unteren Hälfte in sich aufnehmen kann. Es entstebt auf diese Weise eine asymmetrische Gastrula, wie sie Fig. 3 c zeigt. An der Umschlagstelle, dem Blastaporus, geht auch hier die äussere Zellschicht der Gastrula in die innere über. Gemeiniglich stellt man sich die Invagination, bei der der untere Theil der Blastula durch die Aufnahme von Nahrungsdotter ausserordentlich über den oberen an Masse überwiegt, so vor, als wiichse der obere Theil um den unteren herum. Bei dieser Auffassung denkt man sich den unteren Theil als todte Masse, die den festen Punkt abgiebt. Man nennt diese Art der Invagination im Gegensatz zu der embolischen die epibolische Invagination; in der That jedoch ist del Vorgang bei beiden Arten der Invagination der gleiche, der Unterschied besteht nur in der Anschauungsweise, welcher von beiden Hälften wir die lebendige Kraft bei der Invagination beimessen. Aller Wahrscheinlichkeit nach wird wohl jede der beiden Seiten, die obere wie die untere, an der Invagination durch Arbeit betheiligt sein.

Der Blastoporus liegt bei dieser Gastrulation nicht in dem Ende einer Ebene, die die Gastrulahöhle in zwei symmetrische Hälften theilen würde, sondern ist von dieser Ebene nach der Seite des geringern Bildungsmaterials hin verschoben, und zwar scheint nach meinen Beobachtungen an Ampbibieneiern der Blastoporus

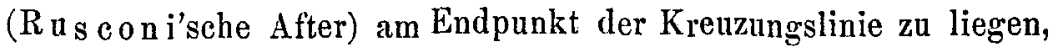
die durch die beiden ersten vertikalen Furchungsebenen gebildet wird, 
von denen, wie oben erwähnt, die erste nicht durch die Mitte des Eies geht. Doch kann ich diese Beobachtung nicht als ganz sicher hinstellen, da nicht alle Fehlerquelleu bei den diesbezüglichen Beobachtungen ausgeschlossen waren; namentlich sind Eigenbewegungen der Eier in ibren schleimigen Hïllen nicht undenkbar.

Wie der Entwicklungsgang aber vielfach verschiedene Wege hat dasselbe Ziel zu erreichen, so finden wir auch bei einer Reihe von Metazoen eine von der Obigen abweichende Dotteraufnabme des Eies. Es kann nämlich das Ei auch den Nahrungsdotter im Centrum um den Kern aufnehmen. Da in diesem Falle jedoch eine Invagination nach der Furchung nicht gut möglich ist, so lagert sich schon im Ei der Bildungsdotter so, dass es nur eines concentrischen Spaltungsprocesses der Blastulazellen mit nachfolgender Trennung derselben an einem Punkt bedarf, um aus der Blastula die Gastrula zu bilden. Diese Art der Gastrulation, die Gastrulation durch Delamination genannt wird, muss man als eine Abkürzung des Bildungsganges auffassen, insofern schon im Ei ein Theil des Bildungsdotters sich in den anderen einstülpt.

Nimmt der Nahrungsdotter immer mehr im Verhältniss zum Bildungsdotter zu, so entrückt er schliesslich der Machtsphäre des Letzteren, d. h. der Bildungsdotter ist nicht mehr im Stande den Nahrungsdotter mit sich zu theilen, zu furchen. Auf diese Weise entsteht die partielle Furchung des Eies. Bei derartigen Eiern findet sich ebenfalls schon im $\mathrm{Ei}$, sei es während oder gar vor der Furchung, eine theilweise Einstiulpung der unteren Bildungsdotterhälfte gegen die obere und es erhält der Bildungsdotter auf diese Weise eine biconvexe Linsenform, die sich der concavconvexen, das ist der Form einer möglichst flach ausgebreiteten Gastrula, nähern kann. Bei diesen Eiern können wir alle drei erwähnten Arten der Gastrulation beobachten. Insofern nämlich erstens der untere Theil des Bildungsdotters sich dem oberen schon vor der Furchung genäbert hat, erhalten wir die Gastrulation durch Delamination, insofern er wäbrend oder unmittelbar nach der Furchung sich dem oberen nähert, baben wir die embolische Gastrulation and schliesslich finden wir auch bei einem Theile dieser Eier mit partieller Furchung die epibolische Invagination. Bei den Eiern nämlich, bei welchen auf der grösseren der beiden asymmetrischen durch die erste Furchungsebene gesetzten Hälften, der Bildungsdotter immerhin im Vergleiche zum Nahrungsdotter 
in hinreichender Menge vorhanden ist (Fig. 6 a), wird noch der Versuch einer epibolischen Gastrulation gemacht, und wir erhalten auf diese Weise auf der einen Seite der Gastrula (der späteren hinteren Seite des Embryo) eine Blastoporuslippe mit einer geringen Einstülpung, einem Rudiment des Blastoporus (Fig. 6b. bl). Bis in die Gastrulahöhle führt dieser Blastoporus nicht, da dieselbe durch den andrängenden Nahrungsdotter zum Theil verstopft ist. Auf dem ganzen übrigen Rande der Gastrula, an dem das Bildungsmaterial in geringerer Masse vorhanden ist, kommt es nicht mehr zur epibolischen Invagination, sondern es wird der Rand der hier auf die beiden zuerst erwähnten Weisen gebildeten Gastrula vom angrenzenden Nahrungsdotter fest zusammen gepresst. Ein sichtbarer Blastoporus ist ebenfalls nicht an diesem Theile des Randes vorhanden; doch geht auch hier wie bei jeder Gastrula die äussere Auskleidung derselben in die innere tiber. Bei den Eiern mit partieller Furchung, bei welchen am ganzen Rande des Keimes der Bildungsdotter in Vergleich zum Nahrungsdotter in zu geringer Menge vorhanden ist, um eine theilweise epibolische Gastrulation za bewirken (Fig. 7 a), wird der Rand des Keimes in seiner ganzen Peripherie durch den angrenzenden Nahrungsdotter zu einer ganz dünnen Platte zusammengedrückt. Es existirt somit auch kein wirklicher Blastoporus oder auch nur ein Rudiment desselben; theoretisch liegt der Blastoports zwischen dem Keimrande und dem angrenzenden Nahrungsdotter. Die Gastrulahöhle ist die sog. Keimhöhle, während vordem die Blastulahöhle durch die sog. Furchungshöhle repräsentirt war.

In das beschriebene Schema passen sämmtliche Eier mit iluren auf die Befruchtung folgenden Entwicklungsstadien; nur die Eier einer Thierklasse machen eine scheinbara Ausnahme, nämlich die Säugethiereier. Dieselben müssten, wie man a priori annehmen sollte, als Eier ohne Nahrungsdotter dem Entwicklungsstypus der Fig. 1 folgen und eine Gastrula durch embolische Invagination bilden; dem ist jedoch nicht so, sondern die Gastrulation dieser Eier gleicht am meisten der Delamination mit gleichzeitiger und nachfolgender Epibolie eines Theiles des Bildungsdotters um einen Hohlraum. Diese Art der Gastrulabildung findet ihre Erklärung in der Annahme, die auch schon von anderer Seite betont ist, dass die Säugethiereier zurückgebildete Eier mit partieller Furchung: seien. Abgesehen von dem verschwundenen Nahrungsdottel sind 
die übrigen Verbältnisse der Säugethiereier dieselben geblieben als die der oben zuletzt beschriebenen Eier mit Nabrungsdotter, so namentlich die Einstiilpung der unteren Bildungsdotterhälfte gegen die obere.

Fig. 5 stellt ein Säugethierei vor, das in einem Stadium dicht vor der gänzlichen Rückbildung des Nahrungsdotters gedacht ist. Der Rand des ursprïnglich linsenförmigen Keimes ist am unteren Theile des Eies verschmolzen. Der Bildungsdotter umfasst noch den letzten Rest vom Nahrungsdotter, oder nach Verschwinden desselben eine Vacuole als Ausdruck des hier noch am wenigsten dichten Bildungsdotters. Wird nun das Ei während oder nach der Furchung durch die Aufnahme von Flüssigkeit bedeutend vergrössert, wie dies beim Säugethierei der Fall ist, so wird die Flussigkeit hauptsächlich dort Aufnahme finden, wo der Nahrungsdotter zuletzt verschwunden ist und wir uns den Bildungsdotter noch am wenigsten dicht vorstellen können. Darch den Druck der Flitssigkeit wird der innere Theil des Bildungsdotters, der noch nicht soweit in der Entwicklung vorangeschritten ist eine zusammenhängende Membran zu bilden, an einer Stelle des schon membranösen äusseren Theiles des Bildungsdotters zusammengedrängt und muss nun von hier aus um den entstandenen Hohlraum herumwachsen (Fig. 5 b).

Wenn ich mir erlauben darf eine Eintheilung der Eier nach den des Längern auseinandergesetzten Gesichtspunkten vorzuschlagen, so wïrde dieselbe zum Theil auf der alten von Remak, zum Theil auf der nenen von Balfour fussen und in folgender Tabelle ihren Ausdruck finden.

Furchung.

I. Holoblastische ${ }^{1}$ ) Eier äqual ... $\left\{\begin{array}{l}\text { symmetrisch. } \\ \text { asymmetrisch. }\end{array}\right.$

II. Meroblastische Eier $\left\{\begin{array}{l}\text { a. total }\left\{\begin{array}{l}\text { telolecithal } \\ \text { centrolecithal }\end{array}\right\} \text { inäqual }\left\{\begin{array}{l}\text { symmetrisch } \\ \text { asymmetrisch }\end{array}\right. \\ \text { b. partiell }\left\{\begin{array}{l}\text { telolecithal } \\ \text { controlecithal (?) }\end{array}\right\} \begin{array}{l}\text { symmetrisch } \\ \text { asymmetrisch. }\end{array}\end{array}\right.$

Aus der dargelegten Auffassung der Gastrula der verschiedenen Metazoen geht hervor, dass ich mich der wohl zuerst von Kupffer vertretenen Anschaung, als entspräche der Primitivstreifen mit der Primitivrinne der Vögel und Reptilien einem Theile

1) $\mathrm{Zu}$ den holoblastischen Fiern sind hier nur Eier ohne Nahrungsdotter gerechnet. 
der Gastrulahöhle beim Amphioxus und den wirbellosen Thieren, nicht anschliessen kann. Und zwar wären meine Gegengründe, ganz kurz gefasst, etwa folgende: Der Primitivstreif ist der optische Ausdruck einer strichförmigen Verdickung des äusseren Keimblattes, auf der bei den meisten Wirbelthieren eine Rinne, die Primitivrinne entsteht, als äusserer Ausdruck einer nach unten stattfindenden Einstülpang dieses Streifens. Diese Bildung bängt einzig und allein mit der Entstehung des Gehirns und Rückenmarks zusammen und findet nicht etwa nur bei den Säugethieren, Vögeln und Reptilien statt, sondern dieselbe tritt bei sämmtlichen Wirbelthieren mit Ausnahme von Lepidosteus, den Teleostiern und Petromyzon auf, bei welchem letzteren die Primitivrinne auf dem Primitivstreifen fehlt, weil sich hier die Hirn- und Rückenmarkshöhle durch einen späteren Spaltungsprocess im Inneren des Primitivstreifens bildet. Beim Amphioxus, von dem $\mathrm{Kupffer}$ in seinen Betrachtungen ausgeht, ist die Primitivrinne darum nicht ohne Weiteres zu sehen, weil die Epidermis zeitlich vor dem Auftreten der Rinne tiber derselben herüberwächst. Auf Schnitten jedoch ist die strichförmige Verdickung mit nachfolgender Einstülpung ebenso wie beim Hühnchen zu sehen. Man kann somit einen beim Amphioxus neben der Gastrulation vorkommenden und von derselben ganz unabhängigen Bildungsprozess bei den anderen Metazoen nicht als Analogon der Gastrulation hinstellen. Bei den Amphibien findet ebenfalls diese strichförmige Verdickung mit nachfolgender Einstïlpung, der auf der Aussenseite einer Rinne entspricht, statt; bei diesen Eiern kann man wegen der Undurchsichtigkeit derselben die Verdickung ebenfalls nur auf Schnitten erkennen.

Ferner möchte ich gegen die Auffassung $K$ upffer's anführen, dass der Keim beim Auftreten der Primitivrinne stets schon zweiblättrig ist, daher durch eine nunmehr auftretende Einstülpung eines Blattes dreiblättrig resp. fünfblättrig werden müsste.

Aus den obigen Auseinandersetzungen uber die Natur des Primitivstreifens geht zugleich hervor, dass ich mich auch der von Balfour vertretenen Ansicht über den Primitivstreifen der Sauropsiden nicht anschliessen kann. Nach seiner Ansicht repräsentirt der Primitivstreifen der Sauropsiden den neurenterischen Theil des Blastoporus der Elasmobranchier, sowie auch den geraden Streifen, der den Elasmobranchierembryo mit dem Blastodermrande 
verbindet. Nach meiner Auffassung entspricht dieser Streifen bei den Ichthyopsiden einem Theile der sog. Narbe (blastotrema K upf fer's) der Keimhaut bei den Sauropsiden und leitet seinen Ursprung daher, dass diese Metazoen den Gastrulationstypus, der in Fig. 6 b dargestellt, einhalten, also an einer Stelle noch eine thatsächliche Invagination zeigen. An dieser Stelle kann der Keim nicht weiter um den Dotter herumwachsen, es bildet sich daher der betreffende Streifen durch das Verschmelzen der seitlich von dieser Stelle befindlichen Keimränder. Den Primitivstreifen dagegen besitzen die Ichthyopsiden, wie oben auseinandergesetzt wurde, ausserdem.

Diese Narbe nun, die sich nach der Umwachsung der Eier durch die Keimhaut am Dotterpole bildet, sei sie nun sternförmig wie bei den Vögeln und Reptilien oder noch ausserdem mit einem Stiele versehen (morgensternförmig) wie bei den Fischen, 'gehört nicht mehr zur Gastrulabildung, sondern ist als eine weitere Entwicklung der schon vollstiindig ausgebildeten Gastrula anzusehen. Diese Bildung entsteht dadurch, dass der Gastrulamund sich schliesst, nachdem el über seine Nahrung herübergewachsen ist. Der Embryo hat inzwischen längst die Phase der Gastrula durchlaufen und ist in dieser Zeit der weitergewachsene Urmundrand zu einer ganz untergeordneten und vorübergehenden Bildung, nämlich zu einem Darmdivertikel herabgesunken.

Es mag hier der Platz sein, auch des oben erwähnten Unterschiedes zu gedenken, der in der Bildung des Hirn- und Rückenmark-Canals der Knochenfische, Lepidosteus und Petromyzon einerseits und der ibrigen Wirbelthiere andererseits besteht; derselbe ist nicht so gross als es auf den ersten. Blick den Anschein hat. Es wird nämlich auch bei den Wirbelthieren, bei denen das Hirn und Rückenmark durch Verschmelzung zweier Wülste nach vorangegangener Rinnenbildung entsteht, der hintere Theil des Rückenmarks, der Schwanztheil desselben derart gebildet, dass sich Zellenmaterial in der Verlängerung des Rückenmarks anhäuft, letzteres auf diese Weise als solider Zapfen weiter wächst, in welchem dann sekundär durch Spaltung von der ursprïnglichen Hirn-Rückenmarkhöhle aus und als Fortsetzung derselben der Ruckenmarkcanal gebildet wird. In sehr grober Weise siebt man diesen Vorgang bei den Amphibien, doch findet derselbe bei sämmtlichen Wirbelthieren statt. Ich bin der Ansicht, dass diese Erscheinung, nämlich die strichförmige Anhäufung von Zellenmaterial 
nach hinten in der Verlängerung des Riickenmarks und ebenso des ursprünglichen Primitivstreifens, insofern diese Anhäufung schon in den juingsten Stadien der Entwicklung beginnt und ungefähr so lange währt, bis das Längenwachstlum des Embryo beendet ist, nichts Anderes ist, als eine Wachsthumserscheinung des Primitivstreifens nach hinten, als erste Anlage des Riickenmarks. Ich habe die Ueberzeugung, dass die Autoren, die der Vorstellung Raum geben, als wiirde der Primitivstreifen nach hinten verschoben, diese Nenbildung des Primitivstreifens vielleicht bei ihren Betrachtungen zu wenig in Rechnung gezogen haben. Abgesehen davon, dass mir eine derartige Verschiebung eines strichförmigen Theiles im Keimblatte physikalisch unmöglich erscheint, wie ich dies schon früher ${ }^{1}$ ) betont habe, möchte ich darauf hinweisen, dass man beim Hühnchen mit ziemlicher Genauigkeit die Lage eines Punktes der Keimscheibe bestimmen und so das Wachsthum eines bestimmten Theiles derselben beobachten kann, indem man ihn nämlich mit dem Rande der area opaca in Vergleich zieht. An diesem Rande, der doch zum grössten Theil von einer todten Masse, nämlich den Nahrungsdotterkïgelchen gebildet wird, sieht man abgesehen von einem gleichmässigen Schwinden dieser Kügelchen in seiner ganzen Peripherie ein besonderes Schwinden des Nabrungsdotters in der Richtung des Primitivstreifens, so dass aus der ursprünglichen Kreisform eine birnenähnliche Form der Peripherie der area opaca resp. der area pellucida wird. Ein Beweis einer besonderen Wachsthumsenergie der hinteren Zellen des Embryo.

Der van Baer'sche Schild mitsammt dem Primitivstreifen beim Reptil, Vogel und Säugethier entspricht nach meiner Auffassung einer ebenso geformten Bildung bei den Amphibien, nämlich der ersten Anlage des Cerebrospinalsystems, die ebenfalls sich der Birnenform nähert. Am verschmälerten Ende dieser Birne geht der Rïckenmarkscanal in das Archenteron uiber und bildet so den neurenterischen Canal. Denken wir uns nun am Ende des Primitivstreifens der Vögel und Reptilien zu einer bestimmten Zeit eine Höhle ${ }^{2}$ ) als Analogon des neurenterischen Canals der Amphi-

1) Archiv f. mikrosk. Anatomie Bd. XXI. Ueber die Keimblätter des Huhnes $\nabla$. Verf.

2) Dass der von Koller beschriebene Sichelknopf etc. vielleicht der Ausdruck dieser heginnenden Canalbildung ist, scheint mir nicht unwahrscheinlich. 
bien entstehen, die bis in das Archenteron hineinreicht, so ist es klar, dass beì dem stetigen Wachsthum, der stetigen Neubildung. von Zellenmaterial am hinteren Ende des Primitivstreifens, alsbald diese Höhle nicht mehr am Ende des Primitivstreifens sich befindet, sondern scheinbar mehr nach vorn rückt. Dazu kommt nocb, dass bei den Vögeln und Reptilien, bei denen der Rand der Gastrula, da an keinem Punkte derselben eine thatsächliche Einstülpung stattgetunden hat, gleichmässig über den Dotter fortwächst, der Primitivstreifen die Möglichkeit hat in der Keimhaut selbst weiter zu wachsen. Da nun ausserdem bei den Vögeln und Reptilien die Gastrula ausserordentlich flach ist, so werden die Niveaunterschiede zwischen dem Primitivstreifen einer'seits und dem neurenterischen Canal andererseits sehr gering, so dass es scheint, als wüchse der Primitivstreifen nicht wie bei den Fischen und Amphibien ïber, sondern hinter dem neurenterischen Canal weiter. Dieses Bild ändert sich erst dann, wenn der Embryo sich über der übrigen Keimhaut erbebt, sich, wie man gewöhnlich sagt, von ihr abschnürt. Vielleicht lassen sich durch die Berlicksichtigung des soeben beschriebenen Wachsthumsvorganges des Primitivstreifens die Widerspritiche der Autoren in Bezug auf die Lage einer Communication der Rückenmarkhöhle mit dem Urdarm bei den Vogelembryonen erklären, insofern nämlich einige Autoren dieselbe vor, andere hinter, endlich andere in die Mitte des Primitivstreifens versetzen. Diese Widersprüche ergeben sich aus dem verschiedenen Alter der untersuchten Embryonen, insofern sich nur zu einer ganz bestimmten Zeit der neurenterische Canal am hinteren Ende des Primitivstreifens befindet. Nach dieser Zeit wächst der letztere nnter fortwährender Umbildung seines vorderen Theiles zum Riickenmark ïber dem netrenterischen Canal nach hinten weiter.

Fassen wir das Wesentliche aus dem beschriebenen Gastrulationsprozess zusammen, so liegt dies in Folgendem: Durch die Gastrulation entwickeln sich aus dem gefurchten Theile zwei zasammenhängende Schichten, von denen die eine in die andere eingestiilpt resp. von der auderen umschlossen wird. Am Umschlagsrande dieser Schichten, der die nach Aussen führende Oeffunng, den Blastoporus umschliesst, geht die äussere Schicht, die wir mit dem Namen äusseres Keimblatt bezeichnen, in die innere, die inneres Keimblatt genannt wird, üher. Von den beiden Keimblättern 
ist das äussere im Wesentlichen das primitive äussere Integument und stellt die schützende empfindende und bewegende Schicht dar, das innere ist im Wesentlichen die primitive innere Auskleidung und stellt die resorbirende und verdanende Schicht vor.

Zwischen diesen beiden Keimblättern treten von den böher entwickelten Coelenteraten an noch Zellen auf, die schlechthin mittleres Keimblatt benannt werden, mögen sie nun eine blattähnliche Anordnung zeigen oder nicht, und zwar sind diese Zellen ontogenetisch entweder vor der Differenzirung des inneren Keimblattes oder gleichzeitig mit derselben gebildet, niemals jedoch entstehen dieselben nach der Ausbildung des inneren Keimblattes. Diese Zellen sind bei den meisten Metazoen nach der Gastrulabildung an der Lippe des Blastoporus gelagert; bei den Wirbelthieren liegen dieselben hauptsächlich an der Seite des Blastoporus, die der späteren Rückenendseite des Embryo entspricht. Man bat nun diese Zellen als sog. mittleres Keimblatt von einem der beiden anderen Keimblätter herleiten zu muissen geglaubt, und zwar hat der grösste Theil der Forscher nach dem Vorbilde Remak's angenommen, dasselbe wäre aus dem inneren Keimblatte hervorgegangen. Diese Annahme beruhte jedoch nicht auf exacten Untersuchungen, sondern war die Frucht einer Spekulation. Es geht nämlich der diploblastische Zustand in der Phylogenie dem triploblastischen voraus. Man bat nun scheinbar berechtigt gefolgert, dass der triploblastische Zustand aus dem diploblastischen entstanden sei, folglich das neu hinzugekommene dritte Keimblatt aus einem der beiden oder den beiden primären Keimblättern hervorgegangen sein muss. Bei dieser Schlussfolge hat man ubbersehen, dass die Keimblätter nicht das primäre sind, sondern der Keim, oder sagen wir kurzweg das Ei, das durch den Furehungsprozess das Material zu den beiden Keimblättern gegeben bat. Sehen wir daher gleichzeitig mit der Bildung der beiden Keimblätter noch einen dritten Zellencomplex sich differenziren, so ist doch wohl der nächstliegendste Gedanke der, diese Zellen direct von den Furchungszellen des Keims abzuleiten und dies um so mehr, als wir diese Zellen häufig schon iın Stadium der Blastosphäre vollständig. unabhängig von den später in die beiden Keimblätter aufgehenden Zellen finden.

In gleicher Weise sehen wir ja auch da, wo das innere Keimblatt uns phylogenetisch zuerst als selbständige Bildung vor 
die Augen tritt, dasselbe sich direct vom gefurchten Keime aus bilden, nicht aber rom äusseren Keimblatte. Wenigstens sehen wir bei Dicyema, das gleichsam das Zwischenglied zwischen den Protozoen and Metazoen bildet, und bei welchem die dem inneren Keimblatte entsprechende Bildnng im ausgewachsenen Zustand des Thieres aus einer einzigen Zelle besteht, dieses innere Keimblatt nicht aus dem änsseren Keimblatte hervorgehen, sondern aus einer Zelle des Keimes, die vorher nicht dem Verbande des äusseren Keimblattes angehört hat. Ich lasse hier die Beschreibung dieses Bildungsprocesses, wie sie Balfour ${ }^{1}$ ) nach den Untersuchungen von van Beneden giebt, folgen:

"Ist der Keim vollständig ausgebildet, so erleidet er eine ganz ähnliche Furchung wie ein gewöhnliches Ei. Er zerfaillt erst in zwei und dann in vier annähernd gleiche Segmente. Von den vier Segmenten bleibt jedoch eines während der ganzen übrigen Entwicklung durchaus passiv. Die anderen drei theilen sich und ordnen sich derartig, dass sie die passive Zelle nach Art eines Bechers theilweise einschliessen. Die aus ihrer Theilung entstandenen sechs Zellen theilen sich dann abermals, so dass zwölf Zellen vorliegen, welche die passive Zelle fast ganz umschliessen, indem nur an einem Punkt eine kleine Oeffung bleibt. Der ganze Vorgang, durch welchen die centrale Zelle eingeschlossen wird, ist, wie E. van Beneden zeigt, identisch mit der Bildung einer Gastrula durch Epibolie und die Stelle, wo die centrale Zelle unbedeckt bleibt, entspricht dem Blastoporus. Letztere geht später in die Hypoblastzelle, die peripherischen Zellen in das Epiblast des ausgewachsenen Thieres uber."

So wenig wie hier das innere Keimblatt aus dem äusseren entstanden ist, so wenig entsteht der Mittelkeim ${ }^{2}$, wie ich diesen Zellencomplex genannt habe, aus dem inneren Keimblatt und alle die Forscher, die denselben vom primären inneren Keimblatte ableiten, beweisen eigentlich nur diese Thatsache, denn in dem Augenblicke, wo aus dem primären inneren Keimblatte sich das sekundäre innere Keimblatt abspaltet, ist auch durch diesen

1) Handbuch der vergleichenden Embryologie von Fr. M. Balfour M. A. F. R. S. ïbersetat von Dr. B. Vetter. Bd. I. 1. Theil. S. 128.

2) o. c. v. V. 
Theilungsprozess der Mittelkeim ${ }^{1}$ ) als Rest des primären inneren Keimblattes gebildet; wir können aber nur das sekundäre innere Keimblatt mit dem inneren Keimblatte der diploblastischen Metazoen in Vergleich ziehen, denn das primäre innere Keimblatt ist doch, wie unmöglich bestritten werden kann, vorläufig nichts anderes als der Keim nach Abzug des äusseren Keimblattes, der weder eine membranöse Form hat, noch auch überhaupt, wie schon mehrfach erwähnt, ganz in die membranöse Form des wirklichen inneren Keimblattes aufgeht.

Vor Allem aber ist auch, so lange noch das sog. primäre innere Keimblatt besteht, die Gastrulaform nicht ausgebildet, insofern die innere membranöse Auskleidung derselben fehlt, deren Bildung auch zugleich, wie ich oben gezeigt habe, das sog. sekundäre innere Keimblatt entstehen lässt.

Wie erwähnt ist der Mittelkeim ein Theil der Furchungszellen, doch geht derselbe, so lange er intact bleibt, d. h. so lange nicht Elemente von einem der beiden Keimblätter in denselben hineingelangt sind, nie eine Lage- oder Formveränderung ein, deren Ergebniss eine membranartige Anordnung seiner Elemente wäre. Ferner betheiligt sich der Mittelkeim nicht an der Gastrulabildung, ist kein integrirender Theil der Gastrula, sondern wird nur als ein Ueberschuss der Furchungselemente, der nicht"zur Bildung der beiden Keimblätter verbraucht ist, $z$ wischen den beiden Blättern der Gastrula beherbergt. Er kann ebenso wie der Nahrungsdotter durch seine Zellenmasse die äussere Form der Gastrula etwas beeinflussen, doch - geht er nicht in einen Bestandtheil der Gastrula über. Aus diesem Grunde babe ich vorgeschlagen, den Namen "mittleres Keimblatt" fallen zu lassen und diesen Zellencomplex Mittelkeim zu nennen. Diese Zurückweisung eines dritten Keimblattes ist nicht unwesentlich, denn nur so wird eine Homologie der Keimblätter bei den gesammten Metazoen ermöglicht.

Man kann sich den Fortschritt in der Differenzirung des Keimes, das Auftreten einer Keimschicht zwischen den beiden Keimblättern, so vorstellen, dass eine Substanz, die in der niederen

1) Er entsteht demnach nicht später als das untere Keimblatt, sondern gleichzeitig mit demselben, ja bei einigen Metazoen ist derselbe suhon im Blastosphärenstaảium vor der Invagination, d. h. also vor dem Process der Keimblattbildung al solcher zu erkennen. 
Reihe der Metazoen in allen Zellen des Keimes vorhanden war, bei den höher entwickelten Metazoen in einem bestimmten Theile desselben ausschliesslich deponirt wird. Dieser Theil geht dann nicht mehr mit den anderen Zellen die Umlagernng in die beiden Keimblätter ein.

Man hat nun nicht allein den Mittelkeim mittleres Keimblatt genannt, sondern bat diese Benennung zugleich auf diesen Zellcomplex übertragen, nachdem Elemente von einem der beiden Keimblätter in denselben hineingelangt waren, er also aufgehört hat ein einheitliches Gebilde zu sein. Es ist aber selbstverständlich eine wissenschaftlicbe Vergleichung der Keimblätter der verschiedenen Metazoen nur dann möglich, wenn man dieselben sich gegenüber: stellt, so lange sie noch einerseits einheitliche, andererseits auch vollständige Gebilde repräsentiren. Denn nachdem eine theilweise Verschmelzung zweier elementarer Keimschichten and in dieser ein Austausch der Elemente stattgefunden hat, wie z. B. im Primitivstreifen der Wirbelthiere zwischen dem äusseren Keimblatte und dem Mittelkeim ein solcher stattfindet, hat die eine Keimschicht soviel an Fähigkeit, Gewebe zu bilden, verloren, als sie Elemente abgegeben hat, wäbrend die andere Keimschicht durch die Aufnahme dieser Elemente ebensoviel an Fähigkeit, Gewebe zu produciren, gewonnen hat und zwar können dies Gewebe sein, die ursprïnglich dieser Keimschicht fremd waren. Ich werde dies an einem concreten Beispiele erläutern.

Bei den Wirbelthieren treten im Primitivstreifen Elemente des äusseren Keimblattes in den Mittelkeim über; nehmen wir nun an, dies wären erstens die sämmtlichen Muskel bildenden Elemente und zweitens ein Theil der Nerven bildenden Elemente, so wäre die Folge davon, wie es in der That der Fall ist, die, dass das äussere Keimblatt der ihm ursprünglich d. h. bei den niederen Metazoen eigenen Fähigkeit muskulöse Elemente za bilden verlustig gegangen ist, während aus der Schicht des Mittelkeimes nun sowohl Muskeln als auch Nerven entstehen; Nerven also vom äusseren Keimblatte und scheinbar auch vom Mittelkeim gebildet werden, Gewebe, die bei den diploblastischen Metazoen aus dem äusseren Keimblatte hervorgehen. In der That aber entstehen auch bei den triploblastischen Metazoen die Muskeln und Nerven nur aus den in den Mittelkeimen eingewanderten Elementen des äusseren Keimblattes, nicht aber aus dem Mittelkeime. 
Diese scheinbare Inconsequenz in der Entstehung der Gewebe aus den embryonalen Keimschichten hat Balfour ${ }^{1}$ ) dureh die Annahme zu erklären versucht, dass durch das Auftreten des sog. mittleren Keimblattes die beiden primären Keimblätter die ibnen ursprünglich zukommende Fïhigkeit gewisse Gewebe zu bilden entweder gänzlich oder theilweise verloren hätten. Andere Forscher, wie Götte, Hiz, Waldeyer, O. und R. Hertwig haben ibberhaupt darauf Verzicht geleistet, die elementaren Keimschichten als die Bildungsstätte bestimmter Gewebe ansehen zu dürfen, und sind zu dem Schlusse gedrängt worden, dass ans jeder Keimschicht, respective jedem Keimblatte fast jedes Gewebe entstehen kann. Man ibersah jedoch, dass man das äussere Keimblatt, nachdem es einen Theil seiner Elemente abgegeben batte, und den Mittelkeim, nachdem derselbe in sich Elemente des äusseren Keimblattes aufgenommen hatte, als intacte Keimblätter ansah und mit solchen in Parallele stellte.

Ich habe oben die Wirbelthiere als ein Beispiel dafür angeführt, dass bei ihnen Elemente des äusseren Keimblattes in den Mittelkeim hineinwandern; diese Einwanderung von Keimblattelementen in den Raum zwischen die beiden Keimblätter, sei nun ein Mittelkeim vorhanden oder nicht, findet jedoch bei allen Metazoen statt, ausgenommen scheinen nur die Hydromedusen, Chaetognathen und vielleicht einige Poriferen. Es scheint mir die Einwanderung von Keimblattelementen in den Raum zwischen die beiden Keimblätter schon in einem niederen Zustande in der Entwicklung der Metazoen aufzutreten als der Mittelkeim, d. h. als Zellen, die nicht von den Keimblättern entstanden, sondern als ein Rest der Furchungselemente übrig geblieben sind; doch lässt sich sehr schwer darüber urtheilen, welche Bildung von diesen beiden in der Phylogenie die frühere ist, da dieselben ziemlich gleichzeitig auftreten und ferner die Autoren in den Resultaten der ontogenetischen Untersuchungen über diesen Punkt auseinanderweichen oder gar kein Gewicht darauf legen, woher die Elemente zwischen den beiden Keimblättern gekommen sind, sondern dieselben ohne Rücksicht auf ihre Entstehung mittleres Keimblatt nennen. Doch bin ich iberzengt, dass allein in der genauen Unterscheidung zwischen den beiden genannten Bildungen in der Axen-

1) o. c. Bd. II. H. 1. p. 311. 
platte ${ }^{1}$ ), wie ich den Mittelkeim bezeichnet habe, nachdem in denselben Elemente vom äusseren Keimblatte (im Primitivstreifen) eingewandert sind, die Möglichkeit gegeben ist, eine Homologie der Keimblätter der Metazoen sowohl in Riicksicht auf ihre Entstehung als auch anf ihre Bedeutung für die Production bestimmter Gewebe zu finden. Bisher ist es aus Grüden, die ich auseindergesetzt habe, den Forschern nicht gelungen, eine Homologie der Keimblätter zu finden, und doch giebt es gewiss sehr wenig Forscher, die glauben, dass eine solche nicht existire, dass eine Form wie die Gastrula, die wir bei den gesammten Metazoen finden, für die Entstehung der Gewebe olne Bedeutung wäre.

Zum Schluss tritt die Beantwortung der Frage an uns heran, welche Bedeutung der Mittelkeim für die Entstehung der Gewebe hat. Die einfacbste Lösung dieser Frage wïrde man finden, wenn es gelänge, die Entwicklung der Elemente desselben bis zu einer gewissen Ausbildung zu verfolgen; leider ist das bis jetzt nicht möglich, da wir vor der Hand kein Mittel besitzen, mit dessen Hülfe wir die Elemente des Mittelkeims von den in ihn hineingewanderten Keimblattelementen im Laufe der Entwicklung nnterscheiden können. Dagegen wissen wir, dass bei einigen Metazoen Zellen des äusseren Keimblattes theilweise sich zn Muskeln umbilden, dass andererseits Muskeln und Nerven aus einer Zelle des äusseren Keimblattes entstehen, dass dagegen die Bindesubstanz als organisirtes Gebilde erst bei den Metazoen auftritt, bei denen ein Mittelkeim vorhanden ist. Es besitzen allerdings Metażoen ohne Mittelkeim eine Stützsubstanz, dieselbe enthält jedoch keine organisirten Gebilde, sie ist nur ein Secret der Keimblattzellen. Es liegt daher sebr nahe anzunehmen, dass der Mittelkeim die Bildungsstätte der organisirten Binde- oder Stiitzsubstanz ist, dass gleichsam die Substanz, die vorher in den Keimblattzellen aufgespeichert war, nun in einem bestimmten organisirten Theile des Keimes, in dem Mittelkeim, deponirt ist.

Das ist der Fortschritt in der Entwicklung, dass nach Auftreten des Mittelkeimes die Keimblattzellen, nun befreit von aller trägen Masse, frei von der Stützsubstanz, sich ausschliesslich ihrem Berufe hingeben können. Ebenso ist es, um ein ähnliches Beispiel anzufïhren, der höchste Grad der Entwickling des Eies, wenn das-

1) Archiv f. mikr. Anat. Bd. XXI. o, c. 
selbe, nachdem es einmal den primitiven Zustand der Bedürfnisslosigkeit verlassen, unbekümmert um seine Nabrung seinen Entwicklungslauf vollenden kann, wie dies in ausgesprochenster Weise beim Säugethiereie der Fall ist. Der Fortschritt in der Entwicklung liegt in dem grossen Princip der Arbeitstheilung.

Vor nicht langer Zeit ist von $O$. und R. Hertwigl) eine eigenartige Theorie über die Entstehung und Deutung der Keimblätter aufgestellt worden. Dieselben suchen von. den Chaetognathen und Brachiopoden ausgehend nachzuweisen, dass bei der einen Abtheilung der Metazoen, zu der sie auch die Wirbelthiere zählen, durch Faltung des Entoblasten zwei neue Keimblätter entstehen, nämlich das parietale und viscerale Mesoblast; dieselben sollen, wie das äussere und innere Keimblatt gleichfalls Schichten epithelial angeordneter Zellen sein, „welche ${ }^{2}$ ) die Flächenbegrenzung des Körpers nach dem neu entstandenen Coelom zu besorgen; sie sind gleichfalls wie die beiden primären Keimblätter durch einen Einfaltungsprozess in das Leben gerufen worden; sie sind auf eine gemeinsame Stammform zu beziehen, deren Urdarm sich durch zwei Falten in drei Räume getheilt hat. Ihre Bildung tritt immer erst nach der Gastrulation ein und deutet einen weiteren Schritt in der Organisation an. Wie die zweiblättrige Gastrula aus der einblättrigen Blastula, so ist aus der zweiblättrigen Gastrula die vierblättrige Coelomform abznleiten." Diese Abtheilung der. Metazoen nennen sie Enterocoelier, welchen die übrigen Metazoen, denen diese beiden neugebildeten Blätter fehlen sollen, als Pseudocoelier gegenübergestellt werden. Das, was ich Mittelkeim genannt habe, wird mit den sich aus dem Verbande der Keimblätter in der weiteren Entwicklung lösenden Zellen zusammengeworfen und als Mesenchym bezeichnet.

Ich kann mich diesem Gedankengange nicht anschliessen, da ich aus logischen Grunden, die ich ausführlich auseinandergesetzt habe, einerseits zwei so heterogene Elemente, wie einestheils der Mittelkeim und anderentheils Elemente, die sich erst in der weiteren Entwicklung von den Keimblättern losgelöst baben, nicht in eine Gruppe zusammenwerfen kann, andererseits auch eine D ifferenzirung eines Theils des Hypoblasts dem Ganzen nicht als eine

1) Die Coelomtheorie von Dr. O. u. R. Hertwig. Jena 1881.

2) o. c. p. 119. 
homologe Bildung an die Seite stellen kann, sondern stets nur als einen Theil desselben betrachten darf, welche Veränderungen derselbe auch später eingehen mag. Nach Hertwig's Gedankengange müssten wir ebenso die Faltung des äusseren Keimblattes in der Primitivrinne der Wirbelthiere als ein pariges neues Keimblatt hinstellen dürfen; ferner könnte man mit demselben Rechte das Amnios auch als zwei neue Keimblätter betrachten, die als eine Faltenbildung aus dem äusseren Keimblatte entstanden sind. Verfolgen wil die Differenzirungen der Keimblätter und des Mittelkeimes weiter, so kommen wir, zumal wir auf die blattförmige Anlage kein besonderes Gewicht legen, annäherud zu den Primitivorganen Reichert's, doch niemals zu neuen Keimblättern.

Von diesen aprioristischen Gründen abgesehen, kann ich die Wirbelthiere (die Vögel, Reptilien und Amphibien nach eigener Untersuchung), wenn ich die von $O$. und R. Hertwig angenommene Bezeichnnng des Coeloms nach seiner Entstehung auf dieselben übertrage, nur für Schizocoelier halten, da sich bei ihnen das Coelom durch einen sekundär auftretenden Spalt in der Axenplatte (dem mittleren Keimblatte der Autoren) bildet, wie es bisher auch wohl sämmtliche Autoren angenommen haben. Speciell kann ich mich mit der Hertwig'schen Auffassung der Entwicklung des Amphibieneies nicht einverstanden erklären, denn einerseits finde ich vor der Bildung der Anlage des Centralnervensystems nirgends im Mesoblasten einen Spalt, der denselben in zwei Blätter theilen könnte; andererseits kann ich auch am Blastoporus weder die Verbindung eines parietalen Blattes des Mesoblasten mit dem äusseren Keimblatte, noch die eines visceralen Blattes mit dem inneren Keimblatte finden, sondern sehe hier nur den continuirlichen Uebergang des äusseren in das innere Keimblatt, welche zusammen den Mittelkeim zwischen sich fassen. Das äussere Keimblatt besteht in dieser Periode aus zwei von einander unterschiedenen Lagen von Zellen, erstens nämlich aus einer einschichtigen Lage pigmentirter grosser Zellen, der sogenannten Umhüllungshaut $R$ eichert's, und zweitens aus einer darunter liegenden mehrschichtigen Lage kleinerer, nicht pigmentirter Zellen; unter diesen Zellen durch einen geringen $Z$ wischenraum von ihnen getrennt, liegt der mehrschichtige Mittelkeim, dessen Bildnng mit der Differenzirung des ihm von unten anliegenden unteren Keimblattes gleichen Schritt hält, vgl. o. c. d. Verf. Taf. V, Fig. 6. 
Mit der Bildung der Rückenfurche verändert sich das Bild beim Froschei gerade so wie beim Hühnerei vollständig und zwar im selbigen Sinne. Der Mittelkeim und das äussere Keimblatt abzïglich der Umhüllungshaut verschwindet von dem Bauchtheile fast vollständig, wie auch schon Götte richtig beobachtet hat, und es sammeln sich die Elemente dieser Gegend um die Ruckenfurche herum, wie Fig. 8 zeigt. Es verschmilzt ferner das äussere Keimblatt abzüglich der Umhüllungshaut (der epithelialen Bekleidung des Embryo) mit dem Mittelkeim zur Bildung der Axenplatte, Fig. 8 x. Ob sich die Anlage des Centralnervensystems und der Chorda schon vor dieser Verschmelzung differenzirt, habe ich vor der Hand nicht entscheiden können; nach Analogie mit dem Huhne wïrden sich diese Anlagen erst aus der Axenplatte ${ }^{1}$ ), wie ich die Combination des Mittelkeins mit den in ihn hineingewucherten Zellen des äusseren Keimblattes genannt habe, bilden. Nach dieser Periode tritt in der peripheren Axenplatte eine Spaltung, die spätere Bauchhöhle, auf, wodurch die Axenplatte sich in das Hautmuskelund Darmfaserblatt trennt, gerade so wie dieser Entwickelungsvorgang bei den Vögeln und Säugethieren vor sich geht; der centrale Theil der Axenplatte gliedert sich in die Urwirbel.

$\mathrm{Ob}$ der durch Faltung des inneren Keimblattes bezüglich der Urdarmwandung entstehende bilaterale Hohlraum um den restirenden Theil des Urdarms der Chaetognathen und Brachiopoden, von denen die Gebrüder Hertwig in ihren Betrachtungen ausgehen, iberhaupt mit der Leibeshöhle der Wirbelthiere verglichen werden kann, ist mir an und für sich sehr zweifelhaft. Wäre es so absurd, wenn man diese Ausstiulpungen des Urdarms der Brachiopoden und Chatognathen mit den Ausstiilpungen respective soliden Auswitchsen des Urdarms der Wirbelthiere, den grossen Darmdrüsen in Parallele stellte? Wahrscheinlicher allerdings scheint mir, dass bei den höheren Metazoen überhaupt kein Analogon dieser paarigen Ausstialpungen des Urdarms der Chaetognathen und Brachiopoden vorhanden ist und dass diese mit dem Genitalapparat in engster Beziehung stehen; bei den Brachiopoden wenigstens scheinen sie nichts weiter als ein Aufbewahrungsort der Eier zu sein, also vielleicht ein paariger Uterus genannt werden zu dürfen.

1) o. c. d. Verf. S. 51. 


\section{Erklärung der Figuren auf Tafel XXVII.}

Fig. 1 a. Holoblastisches Ei mit symmetrischer Furchung. Fig. 1 b. Blastula desselben. Fig. 1 c. Gastrula desselben.

Fig. 2 a. Meroblastisches telolecithales Ei mit totaler symmetrischer Furchung. Fig. 2. Dasselbe schematisch gezeichnet. Fig. 2 b. Blastula desselben. Fig. 2 c. Gastrula desselben.

Fig. 3 a. Meroblastisches telolecithales Ei mit totaler asymmetrischer Furchung. Fig. 3. Dasselbe Ei schematisch gezeichnet. Fig. $3 \mathrm{~b}$. Blastrala desselben. Fig. 3 c. Gatrula desselben.

Fig. 4 a. Meroblastisches centrolecithales Ei mit symmetrischer Furchung. Fig. 4 b. Blastula desselben. Fig. 4 c. Gastrula desselben.

Fig. 5 a. Holoblastisches Ei mit asymmetrischer Furchung. Fig. 5. Dasselbe $\mathrm{Ei}$ als ein fast vollständig zurückgebildetes meroblastisches telolecithales Ei mit partieller Furchung gedacht. Fig. 5 b. Gastrula desselben nahezu vollendet.

Fig. 6 a. Meroblastisches telolecithales Ei mit partieller asymmetrischer Furchung und Einstülpung des unteren Bildungsdotters während der Furchung. Fig. 6 b. Gastrula desselben nahezu vollendet.

Fig. 7 a. Meroblastisches telolecithales Ei mit partieller asymmetrischer Furchung und Einstülpung des unteren Bildungsdotters vor der Furchung. Fig. 7 b. Gastrula desselben.

Fig. 8. Querschnitt eines Froschembryo im Stadium der Hirn-Rückenmarkbildung.

Der Nahrungsdotter ist dunkel, der Bilc̈ungsdotter hell gezeichnet. h. Blastulahöhle (Furchungshöhle). a. Archenteron (Keimhöhle). bl. Blastoporus (prostoma). ae. äusseres Keimblatt. i. inneres Keimblatt. na. Nahrungsdotter. „bi. Bildungsdotter. m. Mittelkeim. x. Axenplatte. prs. Primitivstreifen. 


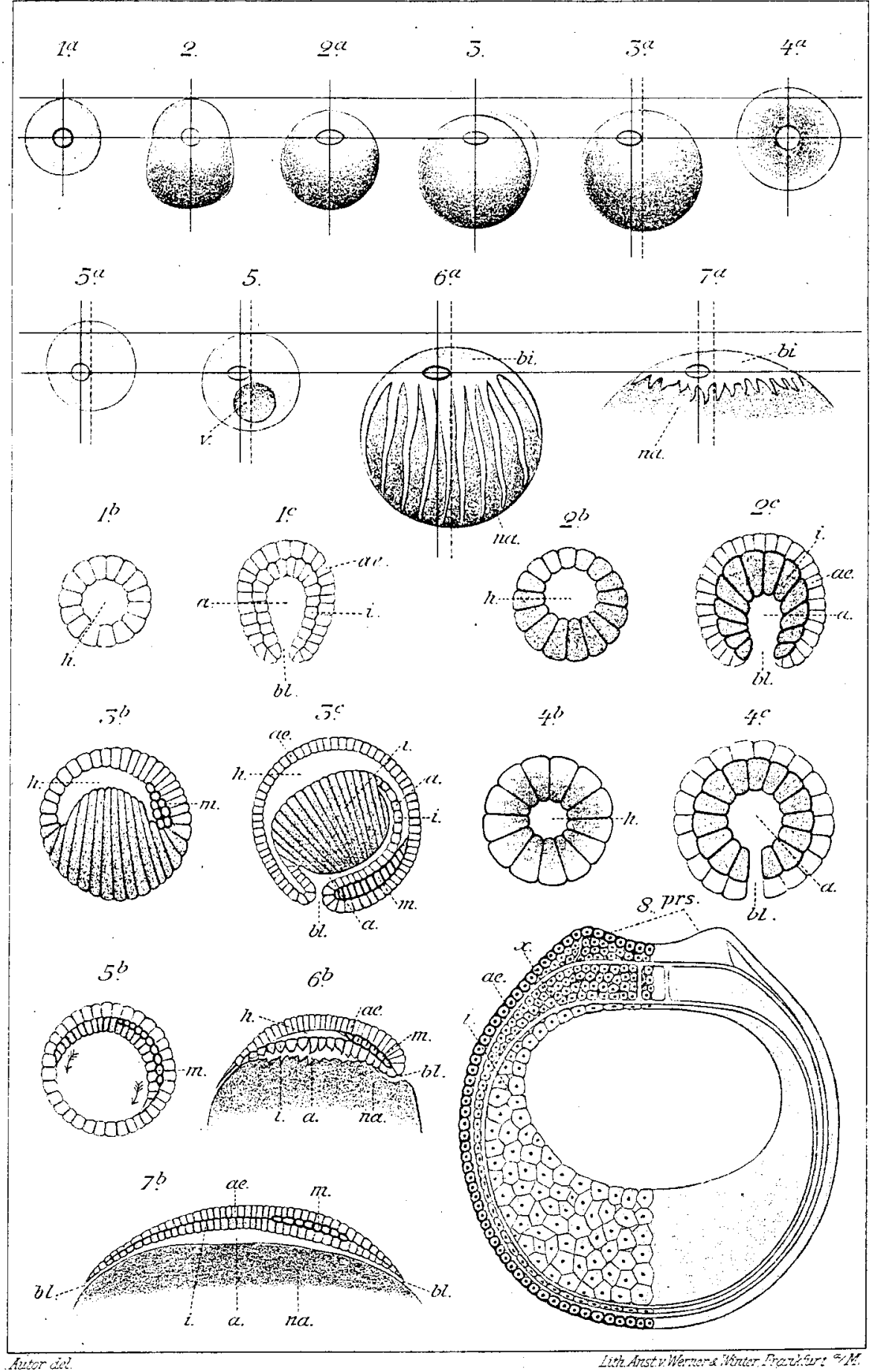

